\title{
DIASTEREOSSELETIVIDADE NA REDUÇÃO DE ENONAS BICÍCLICAS COM HIDRETOS VOLUMOSOS
}

\author{
Andreza C. Camozzato, Beatriz S. M. Tenius e Eduardo R. de Oliveira* \\ Departamento de Química Orgânica, Instituto de Química, Universidade Federal do Rio Grande do Sul, CP 15003, $91501-970$ \\ Porto Alegre - RS, Brasil \\ Cláudio Viegas Jr. \\ Departamento de Farmácia, Universidade Federal de Alfenas, Rua Gabriel Monteiro da Silva, 714, 37130-000 Alfenas - MG, Brasil \\ Maurício M. Victor \\ Departamento de Química Orgânica, Instituto de Química, Universidade Federal da Bahia, Campus de Ondina, 40170-115 \\ Salvador - BA, Brasil \\ Leandro G. da Silveira \\ Departamento de Ciências Exatas e da Terra, Curso de Química, Universidade Regional Integrada do Alto Uruguai e das \\ Missões - Campus de Frederico Westphalen, CP 184, 98400-000 Frederico Westphalen - RS, Brasil
}

Recebido em 14/1/08; aceito em 4/3/08; publicado na web em 23/4/08

\begin{abstract}
DIASTEREOSELECTIVITY IN THE REDUCTION OF BICYCLIC ENONES WITH HINDERED HYDRIDES. Reduction of five substituted octalones employing lithium tri-sec-butylborohydride ( $L$-selectride $\left.{ }^{\circledR}\right)$ in THF and ethyl ether led to allylic alcohols with moderate diastereoselectivity. The stereoselectivity of addition of bulky hydrides showed to be different from most examples in the literature and was strongly influenced by substitution on the octalone ring.
\end{abstract}

Keywords: octalones; reduction; $L$-selectride ${ }^{\circledR}$.

\section{INTRODUÇÃO}

Este artigo é dedicado à memória da Prof ${ }^{a}$. Helena Maria Carvalho Ferraz, que foi inspiradora da linha de pesquisa de síntese de sesquiterpenos em nosso laboratório, com o desenvolvimento da síntese da corimbolona, iniciada na década de $80 .{ }^{1}$ A partir dos anos 90 , temos apresentado uma série de resultados de síntese, ${ }^{2-6}$ de análise espectroscópica ${ }^{7}$ e de estudos mecanísticos ${ }^{8,9}$ envolvendo a química de sesquiterpenos, e todos eles, de certa maneira, foram influenciados pelos trabalhos da Prof ${ }^{a}$. Ferraz. Em nosso trabalho na síntese de sesquiterpenos eudesmanos oticamente ativos e seus derivados, relatamos anteriormente uma eficiente preparação estereosseletiva das octalonas 1 e $4 .^{2}$ Nosso método de preparação do composto 4 mostra-se até hoje como o mais eficiente relatado na literatura e tem sido utilizado para a obtenção de blocos de construção úteis na síntese de muitos e variados produtos naturais, por nós ${ }^{3}$ e por outros. ${ }^{10}$ $\mathrm{Na}$ seqüência de nossos trabalhos sintéticos, temos a necessidade da preparação diastereocontrolada do álcool alílico pseudoaxial $\mathbf{9 b}$. Este composto pode ser obtido pela redução da 14-nor- $\alpha$-ciperona (4) em 2 etapas, com inversão de configuração do álcool. Temos contudo a necessidade de um método de redução direta de $\mathbf{4}$ para $\mathbf{9 b}$.

Nos últimos 30 anos, têm sido muito estudados e relatados na literatura métodos de redução estereocontrolada de cetonas cíclicas e cetonas cíclicas $\alpha, \beta$-insaturadas. Hidretos pouco volumosos como $\mathrm{NaBH}_{4}, \mathrm{LiAlH}_{4}$ e derivados foram avaliados exaustivamente, ${ }^{11-16}$ bem como metais alcalinos em solução. ${ }^{17}$ Resultados experimentais envolvendo cicloexanonas e cicloexenonas substituídas como substratos demonstraram que a direção preferencial do ataque ao anel (axial ou equatorial) possui forte dependência tanto do volume do hidreto atacante quanto do volume dos substituintes ao redor da carbonila. Hidretos pouco volumosos, como $\mathrm{NaBH}_{4}$ e $\mathrm{LiAlH}_{4}$, preferem quase exclusivamente ataques axiais, gerando álcoois pseudoequatoriais

*e-mail: eroliv@iq.ufrgs.br com diastereosseletividades variando de 72 a $>96 \% .^{12-16}$ Os modelos estereoquímicos mais aceitos para explicar esta preferência por um ataque axial são baseados em conceitos como o controle pela estabilidade do produto, e em uma abordagem estérica, pelos efeitos dos hidrogênios $\alpha$-carbonila axiais, que podem repelir e/ou desenvolver efeito torsional quando de ataques equatoriais, e pelos efeitos estéricos dos hidrogênios $\beta$-carbonila axiais (Figura 1). Estudos computacionais ${ }^{18-25}$ confirmaram que tanto efeitos eletrônicos quanto torsionais estão estreitamente relacionados à seleção facial na redução do sistema cicloexanona. A melhor orientação antiperiplanar do nucleófilo com a ligação C-H adjacente em um ataque axial leva a uma deslocalização hiperconjugativa da ligação $\mathrm{C} \alpha-\mathrm{H}_{a x}$ para um ataque axial e da ligação $\mathrm{C} \alpha-\mathrm{C} \beta$ para um ataque equatorial. ${ }^{19}$
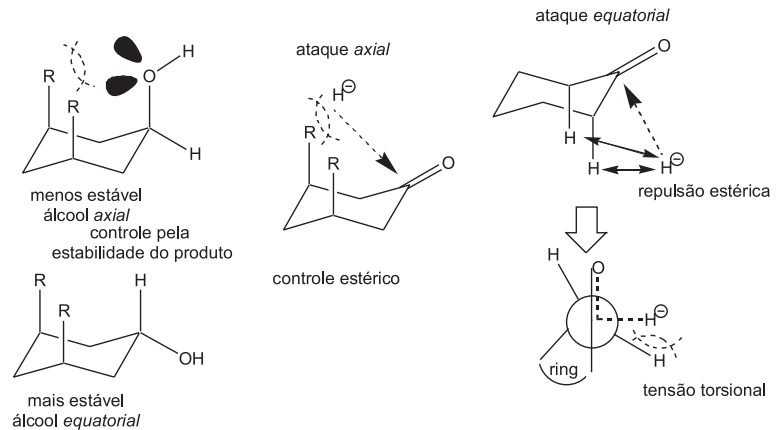

Figura 1. Redução de cicloexanonas. Aspectos estéricos e estereoeletrônicos

Hidretos volumosos, como hidreto de diisobutilalumínio (DIBALH), hidreto de tert-butoxialumínio, ${ }^{26} n$-butilboroidreto de lítio ${ }^{27}$ e trisec-butilboroidretos de lítio e potássio $\left(L\right.$ e $K$-selectride ${ }^{\circledR}$, respectivamente) têm sido investigados na redução de cetonas mono e bicíclicas alquil-substituídas. O DIBAL-H é um dos reagentes mais versáteis para a preparação de álcoois alílicos a partir de enonas. Apesar dos altos 
rendimentos, as misturas de cicloexanóis resultantes contêm preponderantemente, mas não exclusivamente, o isômero pseudo-equatorial..$^{28}$ Os melhores resultados foram obtidos por Saito e colaboradores ${ }^{29}$ na redução de $(4 \mathrm{a} S, 8 S)$-4,4a,5,6,7,8-hexaidro-4a,8-dimetil-2(3H)naftalenona ao $\alpha$-álcool correspondente com $e d=94 \%$, empregando DIBAL-H em uma mistura de THF-DME a baixas temperaturas. Misturas epiméricas também foram obtidas pela redução de 1-etilenodioxi5,5,9-trimetil- $\Delta^{4}$-6-decalona com tri-tert-butoxialuminioidreto de lítio. ${ }^{30}$ Entretanto, trialquilboroidretos mais volumosos mostraram-se capazes de se adicionar a enonas cíclicas e bicíclicas gerando exclusivamente redução 1,2 e, mais importante ainda, por um caminho estereoquímico que fornece o álcool axial, menos estável, como produto majoritário, através de um ataque equatorial preferencial. A prevalência de ataques equatoriais em cetonas cíclicas pode ser atingida tanto pelo aumento do impedimento estérico causado pelos substituintes axiais localizados em C3 e C5 como por aumento do tamanho do reagente hidreto. ${ }^{14,16}$ Entre os agentes redutores extremamente volumosos, os tri-sec-butilboroidretos como $L$-selectride ${ }^{\circledR}$ e $K$-selectride ${ }^{\circledR}$ normalmente produzem excelentes resultados, em preferência direcional totalmente reversa, na qual o ataque equatorial é quase exclusivamente favorecido para todas as cicloexanonas. Estes reagentes foram preparados pela reação de tri-secbutilborano com hidretos de lítio ${ }^{31,32}$ ou potássio. ${ }^{33,34}$ Os autores relataram resultados referentes à sua reatividade, estereosseletividade e aplicabilidade em síntese orgânica. $\mathrm{O} L$-selectride ${ }^{\circledR}$ reduz cetonas cíclicas como 2-metilcicloexanona, 4-tert-butilcicloexanona e 2-metilciclopentanona aos álcoois axiais correspondentes, com diastereosseletividades superiores a $96 \% .{ }^{31} \mathrm{Na}$ redução de derivados de cicloexanona com tri-sec-butilboroidretos, a estereosseletividade está bem esclarecida, tanto experimental ${ }^{16}$ como teoricamente. ${ }^{18-25}$

A redução de cicloexenonas com tri-sec-butilboroidretos já tem sido bastante estudada, ${ }^{35-37}$ principalmente no que se refere à regiosseletividadade. A seletividade das reduções $(1,2$ versus 1,4$)$ pode ser modificada por fatores estéricos, evidenciados pelo favorecimento da redução 1,2 à medida que um impedimento estérico próximo do sítio de reação diminui. ${ }^{36,37}$ Assim como observado para $\mathrm{NaBH}_{4}$, a adição de sais metálicos, como $\mathrm{MgBr}_{2}$ e ZnI , pode influenciar a seletividade de $L$-selectride ${ }^{\circledR}$, que também pode reagir via um complexo intermediário quelado, favorecendo os produtos de redução equatorial. ${ }^{38}$ Mas apesar destes estudos sobre a redução de cicloexenonas com tri-sec-butilboroidretos ainda existe uma lacuna nos dados de estereosseletividade, que não estão completamente elucidados e generalizados, e que são o objeto deste trabalho.

\section{RESULTADOS E DISCUSSÃO}

Neste artigo relatamos nossos resultados na redução das enonas bicíclicas 1-5, com $L$-selectride ${ }^{\circledR}$. Paralelamente, reduções com $\mathrm{LiAlH}_{4}$ foram realizadas, para efeitos de comparação. Como no caso dos análogos saturados, esperava-se que o impedimento estérico de um hidrogênio axial em C-1 levaria preferencialmente ao ataque equatorial, que resultaria nos álcoois alílicos pseudoaxiais 6b-10b (Esquema 1).

Como mostrado na Tabela 1, as reações com $\mathrm{LiAlH}_{4}$ forneceram os álcoois alílicos pseudoequatoriais 6a-10a correspondentes, com boa diastereosseletividade (entradas 1-5), da mesma forma que com DIBAL-H $,{ }^{29} \mathrm{Li} n \mathrm{BuBH}_{3},{ }^{27}$ ou $\mathrm{Li}(\mathrm{O} t \mathrm{Bu})_{3} \mathrm{AlH} .{ }^{39}$ Por outro lado, a redução com $L$-selectride ${ }^{\circledR}$ dos mesmos compostos produziu misturas dos álcoois alílicos epiméricos 6-10 em diferentes proporções (entradas 6-10). Esperava-se que os fatores estérico e estereoeletrônico competissem durante a aproximação do hidreto ao grupo carbonila, na presença de substituintes volumosos em posições remotas, como a isopropenila em $\mathrm{C}-7$, agindo em conjunto com o grupo metila axial na cabeça-de-ponte. $\mathrm{O}$ átomo de hidrogênio axial em $\mathrm{C}-1$ pode ter
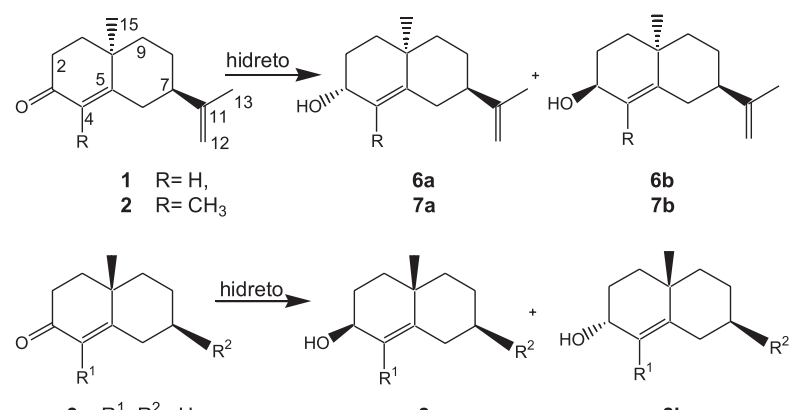

$3 \quad R^{1}=R^{2}=H$

$4 \mathrm{R}^{1}=\mathrm{H}, \mathrm{R}^{2}=-\mathrm{C}\left(=\mathrm{CH}_{2}\right) \mathrm{CH}_{3}$

$5 \mathrm{R}^{1}=\mathrm{CH}_{3}, \mathrm{R}^{2}=-\mathrm{C}\left(=\mathrm{CH}_{2}\right) \mathrm{CH}_{3}$,
$8 a$

$9 a$

$10 \mathrm{a}$
$8 b$

96
Esquema 1. Reações de redução dos compostos 1-5

um papel determinante na aproximação do hidreto volumoso pelo lado oposto àquele observado com hidretos pequenos. Se examinarmos o composto 3, na redução com $\mathrm{LiAlH}_{4}$ (entrada 3), a preferência por um ataque axial é clara, em um controle estereoeletrônico evidente. Entretanto, observa-se na redução com $L$-selectride ${ }^{\circledR}$ (entrada 8) uma preferência crescente pela trajetória equatorial do hidreto, como esperado pelo controle estérico para este tipo de reação, ainda que o ataque axial continue sendo majoritário. Este resultado é o oposto do observado para a redução de cetonas saturadas, que leva majoritariamente aos álcoois axiais. É importante citar que nas descrições prévias da redução de $\mathbf{3}$ com $L$-selectride ${ }^{\circledR}$, os dados de $e d$ não foram fornecidos pelos autores, ${ }^{35,36}$ que descreveram apenas a regiosseletividade $1,2(>95 \%)$, da mesma forma que o observado neste trabalho. Neste caso, a presença de um hidrogênio com orientação axial em C-1 parece ser importante. Contudo, a ausência de outro hidrogênio axial em C-5, como em uma cetona saturada, conduz a um ataque axial ainda predominante.

Como pode ser visto nas entradas 6-10, em nenhum caso observa-se uma preferência por um ataque equatorial, como poderia ser esperado, por analogia com cetonas saturadas. Ao contrário, a preferência por ataque axial é verificada na maioria dos casos.

Tabela 1. Dados experimentais da redução das enonas bicíclicas 1-5

\begin{tabular}{|c|c|c|c|c|}
\hline Entrada & enona & hidreto & $\begin{array}{c}\text { Seletividade } \\
\text { (a:b) }\end{array}$ & $\begin{array}{l}\text { Tempo de } \\
\text { reação }(h)\end{array}$ \\
\hline 1 & 1 & $\mathrm{LiAlH}_{4}$ & $9: 1$ & $2^{\mathrm{a}}$ \\
\hline 2 & 2 & $\mathrm{LiAlH}_{4}^{4}$ & $5: 1$ & $5^{\mathrm{a}}$ \\
\hline 3 & 3 & $\mathrm{LiAlH}_{4}$ & $9: 1$ & $2^{a}$ \\
\hline 4 & 4 & $\mathrm{LiAlH}_{4}$ & $9: 1$ & $2^{\mathrm{a}}$ \\
\hline 5 & 5 & $\mathrm{LiAlH}_{4}$ & $3.5: 1$ & $5^{\mathrm{a}}$ \\
\hline 6 & 1 & $L$-selectride ${ }^{\circledR}$ & $1: 1$ & $2^{\mathrm{b}}$ \\
\hline 7 & 2 & $L$-selectride ${ }^{\circledR}$ & $1: 1$ & $5^{c}$ \\
\hline 8 & 3 & $L$-selectride ${ }^{\circledR}$ & $5: 1$ & $2^{\mathrm{b}}$ \\
\hline 9 & 4 & $L$-selectride ${ }^{\circledR}$ & $2: 1$ & $2^{\mathrm{b}}$ \\
\hline 10 & 5 & $L$-selectride ${ }^{\circledR}$ & $1: 1$ & $5^{\mathrm{b}}$ \\
\hline
\end{tabular}

a. $\mathrm{Et}_{2} \mathrm{O}, 0{ }^{\circ} \mathrm{C}, 1,3$ eq. $\mathrm{LiAlH}_{4} ; b$. THF, $-78{ }^{\circ} \mathrm{C}, 1,2$ eq. $L$-selectride ${ }^{\circledR}$

Para tentar compreender o decréscimo na proporção do ataque axial na ordem 3>4>1 (entradas 8, 9 e 6 , respectivamente), decidimos calcular as geometrias destes substratos, para a determinação da posição preferencial do grupo isopropenila em relação à trajetória do nucleófilo. Utilizando o programa Gaussian $98,{ }^{40}$ iniciou-se por uma otimização preliminar das geometrias com método semiempírico AM1, seguida pelo cálculo $a b$ initio RHF, com base 6$311 \mathrm{G}(\mathrm{d}, \mathrm{p})$ para obtenção das estruturas finais. Pela análise dos dados da Tabela 2, verifica-se que para todos os compostos 1-5 a confor- 
mação mais estável para o ciclo B é a cadeira, com a isopropenila em posição equatorial para a 14-nor- $\alpha$-ciperona (4) e para a $\alpha$ ciperona (5) (entradas 4 e 5), o que era esperado. Calculamos vários confôrmeros limitantes com rotação em torno da ligação C-7$\mathrm{C}_{\mathrm{sp} 2}(\mathrm{C}-11$ isopropenila), mas as diferenças de energia não foram significativas (menores que $K \mathrm{~T}$ ).

A diferença conformacional entre as geometrias cadeira e barco pode ser estimada em cerca de $5 \mathrm{kcal} / \mathrm{mol}$, como determinado para a octalona 3 (entrada 3 ), onde o ciclo $B$ não possui substituintes. Esta diferença de energia aumenta no caso das octalonas cis $\mathbf{4}$ e $\mathbf{5}$, pela tensão 1,4-diaxial que existe no confôrmero com a isopropenila axial. Já no caso das octalonas trans 14-nor-10-epi- $\alpha$-ciperona (1) e 10-epi- $\alpha$-ciperona (2), onde os confôrmeros mais estáveis apresentam o substituinte isopropenila em posição axial, ao contrário do que se poderia supor, a diferença de energia entre os confôrmeros limitantes é menor, como mostrado nas entradas 1 e 2 . Há nestes casos uma competição entre a tensão torsional desenvolvida em uma conformação barco torcido e a tensão 1,4-diaxial que se cria com a adoção de uma conformação axial pela isopropenila quando o ciclo B está na conformação cadeira. Este resultado foi anteriormente descrito por Huffman e colaboradores, utilizando técnicas de dicroísmo circular e RMN com indução de deslocamento por lantanídeo, ${ }^{41}$ o que é confirmado por nossos cálculos. ${ }^{42}$ As estruturas mais estáveis para as octalonas 1-5 estão disponíveis como Material Suplementar, na Figura 1S, e suas respectivas energias estão descritas na Parte Experimental.

Tabela 2. Dados de diferença de energia entre os confôrmeros limitantes do ciclo B das octalonas $\mathbf{1 - 5}$

\begin{tabular}{lcccc}
\hline Entrada & octalona & $\begin{array}{c}\text { Confôrmero } \\
\text { cadeira }\end{array}$ & $\begin{array}{c}\text { Confôrmero } \\
\text { barco }\end{array}$ & $\begin{array}{c}\Delta \Delta \mathrm{H}_{\mathrm{f}} \\
(\mathrm{kcal} / \mathrm{mol})\end{array}$ \\
\hline 1 & 1 & $1 \mathrm{ca}$ & $1 \mathrm{ba}$ & 3,15 \\
2 & 2 & $2 \mathrm{ca}$ & $2 \mathrm{ba}$ & 2,10 \\
3 & 3 & $3 \mathrm{ca}$ & $3 \mathrm{ba}$ & 5,47 \\
4 & 4 & $4 \mathrm{ca}$ & $4 \mathrm{ba}$ & 8,71 \\
5 & 5 & $5 \mathrm{ca}$ & $5 \mathrm{ba}$ & 7,94 \\
\hline
\end{tabular}

${ }^{a}$ Diferença de energia entre os confôrmeros de menor energia em cada caso (cadeira-barco)

Outra evidência da conformação barco torcido para as octalonas 1 e 2 foi obtida pelos espectros de RMN ${ }^{13} \mathrm{C}$, como mostra a Tabela 3..$^{2,3,41}$ Os carbonos C-9 em $\mathbf{1}$ e $\mathbf{2}$ apresentam uma importante blindagem em seus deslocamentos químicos, se comparados com os correspondentes valores para 4 e 5. Este efeito deriva de uma interação $\gamma$-gauche entre a isopropenila axial e C-9. Os ângulos diedros calculados para os confôrmeros 1 ca e 2 ca foram de cerca de $75^{\circ}$ entre o carbono central da isopropenila (C-11) e C-9, como mostrado na Figura 1S (Material Suplementar), para os confôrmeros 1ca e 2ca. Igualmente, C-7 e C-8 apresentam blindagens em seus deslocamentos químicos quando a isopropenila está em conformação axial, como também pode ser visto na Tabela 3, onde são mostrados igualmente os dados de deslocamentos químicos de RMN ${ }^{13} \mathrm{C}$ para os álcoois epiméricos $9 \mathbf{a}$ e $\mathbf{9 b}$, que como as octalonas $4 \mathrm{e}$ 5 apresentam a isopropenila em posição equatorial. Um dado interessante que pode ser extraído da Tabela 3 é uma curiosidade observada pela Prof ${ }^{a}$. Helena M. C. Ferraz, em comunicação pessoal. A soma dos valores de deslocamento químico dos carbonos $\mathrm{sp}^{2} \mathrm{da}$ isopropenila (C-11 e C-12) é sempre cerca de 258 ppm, independente da estereoquímica.

Ainda dentro do objetivo de confirmar esta posição axial para a isopropenila, nós utilizamos técnicas avançadas de $\mathrm{RMN}{ }^{1} \mathrm{H}(\mathrm{J}-$
Tabela 3. Dados de RMN ${ }^{13} \mathrm{C}$ das octalonas e produtos de redução

\begin{tabular}{lccccccc}
\hline & \multicolumn{3}{c}{$\delta$ (ppm) } & \multicolumn{3}{c}{$\begin{array}{c}\text { Soma } \delta \\
(\mathrm{ppm})\end{array}$} \\
composto & C-7 & C-8 & C-9 & C-11 & C-12 & $\begin{array}{c}\text { C-11+ } \\
\text { C-12 }\end{array}$ & Ref. \\
& & & & & & & \\
\hline $\mathbf{1}$ & 40,2 & 23,4 & 35,3 & 146,6 & 112,0 & 258,6 & 2 \\
$\mathbf{2}$ & 41,1 & 23,2 & 36,0 & 147,6 & 111,2 & 258,8 & 3,41 \\
$\mathbf{4}$ & 46,0 & 27,0 & 41,1 & 148,4 & 109,2 & 257,6 & 2 \\
$\mathbf{5}$ & 46,0 & 27,0 & 42,0 & 149,1 & 109,4 & 258,5 & 3,41 \\
9a & 47,4 & 27,1 & 41,7 & 149,8 & 108,5 & 258,3 & $a$ \\
9b & 46,9 & 27,3 & 41,3 & 149,8 & 108,5 & 258,3 & $a$ \\
\hline
\end{tabular}

${ }^{a}$ Dados deste trabalho

resolvido homonuclear - HOM2DJ), na medida em que não é possível uma análise de primeira ordem dos sinais dos hidrogênios H2, H-7 e H-6, que absorvem todos em um multipleto não resolvido entre 2,76 e 2,29 ppm a $200 \mathrm{MHz}$ para o composto 1. Na Figura 2S, Material Suplementar, mostra-se o espectro de HOM2DJ para esta seção do espectro de $\mathrm{RMN}{ }^{1} \mathrm{H}$, onde é possível obter-se os valores das constantes de acoplamento para estes 5 hidrogênios (sumarizados na Tabela 4). A partir destes dados pode-se concluir que o hidrogênio H-7 se apresenta em posição equatorial, de sorte que a isopropenila está em posição axial, análise consistente com uma conformação cadeira para o ciclo B.

Tabela 4. Dados das constantes de acoplamento para H-2, H-6 e $\mathrm{H}-7$, obtidas no espectro de RMN HOM2DJ para o composto $\mathbf{1}$

$\delta$ (ppm) Constantes de acoplamento observadas (Hz) Atribuição $2,67 \quad J=16, J^{\prime}=7, J^{\prime \prime}=2 \quad J_{\text {gem }}, J_{\text {ax -eq }}, J_{\text {eq-eq }} \quad \mathrm{H} 2_{\text {eq }}$ $\begin{array}{llll}2,56 & J=10, J^{\prime}=7 & J_{\text {eq-ax-eq }}, J^{\text {eq-eq }} & \mathrm{H} 7\end{array}$

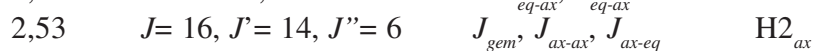
$2,51 \quad J=17, J^{\prime}=2 \quad J_{g e m}, J_{W}{ }^{a x-e q} \quad \mathrm{H6}_{e q}{ }^{a x}$

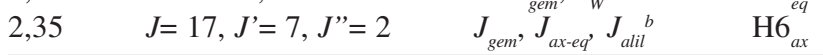

${ }^{a}$ Acoplamento em W com H- $8_{e q}{ }^{41}{ }^{b}$ Acoplamento alílico com H-4.

Conhecendo-se a estereoquímica dos substratos, pode-se então fazer uma racionalização dos resultados das reduções com $L$ selectride ${ }^{\circledR}$, apresentados na Tabela 1. Observa-se que os substituintes em C-4 e C-7 no anel das octalonas causam um efeito importante tanto sobre a estereosseletividade quanto na reatividade da adição. Quando as octalonas são substituídas em C-4 (entradas 2, 5, 7 e 10), as reações requerem mais tempo para se completar. Tal resultado pode ser atribuído a um aumento no impedimento estérico na região da carbonila. Contudo, a quimiosseletividade da reação não se altera, e observam-se exclusivamente os produtos de adição 1,2. É interessante notar que a metila em C-4 causa uma diminuição da estereosseletividade da redução com $\mathrm{LiAlH}_{4}$ (entradas 2 e 5), que talvez seja devida à menor velocidade da reação.

Já a influência do grupo isopropenila em C-7 sobre o ataque do hidreto é fortemente dependente da estereoquímica relativa deste centro. Tal influência pode ser comprovada comparando-se a diastereosseletividade da redução por $L$-selectride ${ }^{\circledR}$ das enonas 1, 4 e 3 (entradas 6, 9 e 8, respectivamente), onde uma preferência crescente pela trajetória axial está associada a um impedimento estérico decrescente na face $\alpha$, como pode ser visto na Figura 2.

Estes resultados mostram que a trajetória da redução de enonas com $L$-selectride ${ }^{\circledast}$ sofre um determinante controle estérico. A ausência de um hidrogênio axial em C-5 diminui dramaticamente a tendência por uma trajetória equatorial, como ocorre com cetonas saturadas. Mas a tendência normal por ataque axial, favorecido tanto estérica 


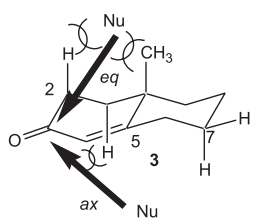

razão ax/eq 5:1

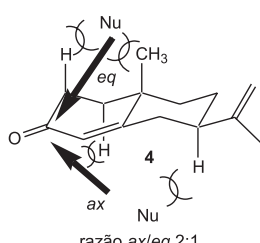

razão axleq 2:1

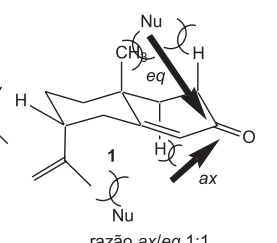

razão axleq 1:1
Figura 2. Impedimento estérico para a redução das enonas com hidretos

quanto estereoeletronicamente, sofre uma influência determinante da presença de substituintes mesmo em posições remotas, como é o caso das octalonas 1 e 4, que apresentam substituintes isopropenila em C7. A presença da isopropenila em posição axial, na octalona $\mathbf{1}$, determinada tanto computacionalmente quanto por RMN, leva a uma diminuição ed de $71 \%$ (entrada 8) para zero (entrada 6) do ed para um ataque axial. Mesmo uma isopropenila em posição equatorial, como na octalona 4, causa impedimento estérico suficiente para diminuir o ed para 33\% (entrada 9). Para os substratos substituídos em C-4, observa-se uma semelhança de resultados entre a octalona 2 (entrada 7) e a octalona 1 (entrada 6). Contudo, a octalona 5 apresenta um ed de zero (entrada 10), ao contrário do ed de 33\%, esperado, por analogia com a enona 4, da mesma forma que ocorre para a redução com $\mathrm{LiAlH}_{4}$.

\section{PARTE EXPERIMENTAL}

Os compostos $\mathbf{1}$ e $\mathbf{2}$ foram preparados de forma enantiosseletiva, de acordo com procedimento descrito. ${ }^{2} \mathrm{O}$ composto $\mathbf{3}$ foi adquirido da Aldrich, e utilizado sem nenhuma purificação. Os compostos 4 e 5 foram preparados conforme metodologia descrita na literatura. ${ }^{3}$ Os reagentes $\mathrm{LiAlH}_{4}$ e $L$-selectride ${ }^{\circledR}$ foram adquiridos da Aldrich, e utilizados sem nenhuma purificação. Os solventes éter etílico e THF foram secos sob sódio metálico e destilados imediatamente antes do uso.

Os álcoois epiméricos 6-10 são inseparáveis em cromatografia em coluna e/ou cromatografia em camada delgada preparativa. Assim, para determinação das proporções relativas, as misturas obtidas foram analisadas em $\mathrm{CG}$ e $\mathrm{RMN}{ }^{1} \mathrm{H}$. $\mathrm{O}$ álcool 9a foi preparado por redução da octalona 4 com $\mathrm{LiAlH}_{4}$ (conforme procedimento a seguir), em rendimento de $98 \%(e d=80 \%)$. Com a metodologia de Mitsonobu 9a foi convertido em 9b, como descrito a seguir.

\section{Procedimento geral para redução com $\mathrm{LiAlH}_{4}$}

A uma suspensão de $\mathrm{LiAlH}_{4}(13,02 \mathrm{mmol})$ em éter etílico seco (40 mL), uma solução de enona $(9,79 \mathrm{mmol})$ em éter etílico seco $(20 \mathrm{~mL})$ foi adicionada a $0{ }^{\circ} \mathrm{C}$. A mistura foi agitada por $2-5 \mathrm{~h}$. Após o término da reação, água $(1 \mathrm{~mL})$ e solução $\mathrm{NaOH}$ 5\% (1,5 $\mathrm{mL})$ foram adicionadas. Após agitação por uma noite, a mistura foi filtrada e o solvente destilado sob pressão reduzida em evaporador rotatório, fornecendo uma mistura de álcoois (rendimento de 95-98\%). A composição da mistura foi determinada por CG e análise de espectros de RMN.

9a. IV (KBr) $v_{\text {max. }} \mathrm{cm}^{-1}: 3300,1640,890 . \mathrm{RMN}^{1} \mathrm{H}(200 \mathrm{MHz}$, $\left.\mathrm{CDCl}_{3}\right) \delta_{\mathrm{H}}: 1,12(3 \mathrm{H}, \mathrm{s}) ; 1,28(2 \mathrm{H}, \mathrm{m}) ; 1,52(6 \mathrm{H}, \mathrm{m}) ; 1,75(3 \mathrm{H}, \mathrm{s})$; $2,10(4 \mathrm{H}, \mathrm{m}) ; 4,22(1 \mathrm{H}, \mathrm{m}) ; 4,72(2 \mathrm{H}, \mathrm{s}) ; 5,34(1 \mathrm{H}, \mathrm{s}) . \mathrm{RMN}-{ }^{13} \mathrm{C}$ $\left(50 \mathrm{MHz}, \mathrm{CDCl}_{3}\right) \delta_{\mathrm{C}}: 20,8(\mathrm{C}-13) ; 23,8$ (C-14); 27,1 (C-8); 29,1 (C-2); 34,5 (C-10); 37,1 (C-1); 37,3 (C-6); 41,7 (C-9); 47,4 (C-7); 68,1 (C-3); 108,5 (C-12); 124,1 (C-4); 145,9 (C-5); 149,8 (C-11).

\section{Preparação do álcool 9b}

Primeira etapa - inversão de configuração

Em balão monotubulado, colocaram-se $975 \mathrm{mg}(4,73 \mathrm{mmol})$ do álcool 9a e $23 \mathrm{~mL}$ de benzeno e adicionaram-se 2,49 g (9,5 mmol) de $\mathrm{Ph}_{3} \mathrm{P}$ e $1,59 \mathrm{~g}(9,5 \mathrm{mmol})$ de ácido $p$-nitrobenzóico. Resfriou-se o sistema a $0{ }^{\circ} \mathrm{C}$ e adicionou-se, lentamente, uma solução de 1,5 mL de DEAD em $4 \mathrm{~mL}$ de benzeno. Deixou-se reagir à t.a. durante $20 \mathrm{~h}$. Filtrou-se e o solvente foi destilado à pressão reduzida em evaporador rotatório. O produto foi purificado em coluna de sílica gel (eluente: hexano/acetato de etila a 1-3\%), fornecendo 1,4 $\mathrm{g}$ do éster derivado de $\mathbf{9 b}$ em $83 \%$ de rendimento, cujos dados espectroscópicos estão descritos a seguir.

IV (filme): $v_{\text {max. }} \mathrm{cm}^{-1} .3080 ; 2940 ; 1700 ; 1640 ; 1600 ; 1540 ; 1360$; $1280 ; 890 ; 750 ; 700 . \mathrm{RMN}^{1} \mathrm{H}\left(200 \mathrm{MHz}, \mathrm{CDCl}_{3}\right) \delta_{\mathrm{H}}: 8,3-8,2(\mathrm{~m}$, $4 \mathrm{CH}$ anel aromático); 5,59 (d, J=4,8 Hz, 1H-3); 5,45 (m, 1H-4); 4,70 (s, 2H-12); 2,18-1,95 (m, 4H); 1,76 (s, 3H-13); 1,74-1,45 (m, $7 \mathrm{H}) ; 1,12$ (s, 3H-15). RMN ${ }^{13} \mathrm{C}\left(50 \mathrm{MHz}, \mathrm{CDCl}_{3}\right) \delta_{\mathrm{C}}: 20,8$ (C-13); 22,9 (C-15); 25,0 (C-2); 27,3 (C-8); 34,6 (C-1); 34,7 (C-10); 37,4 (C-6); 41,4 (C-9); 46,7 (C-7); 69,9 (C-3); 108,7 (C-12); 117,3 (C-4); $123,4\left(2 \mathrm{CH} m-\mathrm{NO}_{2}\right) ; 130,7\left(2 \mathrm{CH} o-\mathrm{NO}_{2}\right) ; 136,2\left(\mathrm{C} p-\mathrm{NO}_{2}\right) ; 149,5$ (C-11); 151,3 (C-5); 164,5 (C=O).

\section{Segunda etapa - hidrólise do éster derivado de $9 b$}

A uma solução de $340 \mathrm{mg}(1,04 \mathrm{mmol})$ do éster derivado de $\mathbf{9 b}$ em $10 \mathrm{~mL}$ de $\mathrm{MeOH}$ e $3 \mathrm{~mL}$ de hexano foram adicionados 2,5 mL de água e $0,80 \mathrm{~g}(5,7 \mathrm{mmol})$ de $\mathrm{K}_{2} \mathrm{CO}_{3} \mathrm{~A}$ reação foi mantida à $\mathrm{t}$. a. por $3 \mathrm{~h}$. Destilou-se o solvente à pressão reduzida em evaporador rotatório e extraiu-se a fase aquosa com acetato de etila $(4 \times 10 \mathrm{~mL})$. Os extratos orgânicos combinados foram lavados com $10 \mathrm{~mL}$ de solução saturada de $\mathrm{NaCl}$ e secos sobre $\mathrm{MgSO}_{4}$ anidro. $\mathrm{O}$ solvente foi novamente destilado à pressão reduzida, fornecendo $196,4 \mathrm{mg}$ do álcool 9 b puro, em $80 \%$ de rendimento.

9b IV (KBr) $v_{\text {max. }} \mathrm{cm}^{-1}: 3300,1650,900$. RMN- ${ }^{1} \mathrm{H}(200 \mathrm{MHz}$, $\left.\mathrm{CDCl}_{3}\right) \delta_{\mathrm{H}}: 1,05(3 \mathrm{H}, \mathrm{s}) ; 1,51(10 \mathrm{H}, \mathrm{m}) ; 1,75(3 \mathrm{H}, \mathrm{s}) ; 2,15(2 \mathrm{H}, \mathrm{m})$; $4,12(1 \mathrm{H}, \mathrm{m}) ; 4,70(2 \mathrm{H}, \mathrm{s}) ; 5,50(1 \mathrm{H}, \mathrm{d}, J 4,5 \mathrm{~Hz}) . \mathrm{RMN}-{ }^{13} \mathrm{C}(50$ $\left.\mathrm{MHz}_{2} \mathrm{CDCl}_{3}\right) \delta_{\mathrm{C}}: 20,8(\mathrm{C}-13) ; 23,0(\mathrm{C}-14) ; 27,3$ (C-8); 28,0 (C-2); 34,1 (C-1); 34,7 (C-10); 37,5 (C-6); 41,3 (C-9); 46,9 (C-7); 64,8 (C-3); 108,5 (C-12); 122,0 (C-4); 148,1 (C-5); 149,8 (C-11).

\section{Procedimento geral para redução com $L$-selectride ${ }^{\circledR}$}

A uma solução de enona $(0,5 \mathrm{mmol})$ em THF seco $(0,5 \mathrm{~mL}), L$ selectride ${ }^{\circledR}(0,6 \mathrm{mmol})$ foi adicionada lentamente à $-78^{\circ} \mathrm{C}$. A solução foi agitada por 2-8 h à temperatura ambiente. Após o término da reação, uma solução de $\mathrm{NaOH} 3 \mathrm{~N}(0,2 \mathrm{~mL})$ foi adicionada e a solução foi mantida em repouso por $30 \mathrm{~min}$. A mistura foi extraída com acetato de etila $(2 \times 10 \mathrm{~mL})$ e destilada sob pressão reduzida em evaporador rotatório, fornecendo uma mistura de álcoois (rendimento 95-98\%). A composição da mistura foi determinada por CG e RMN.

O estudo teórico das octalonas 1-5 foi realizado com o objetivo de elucidar as propriedades estruturais dos confôrmeros mais estáveis. As energias dos confôrmeros estudados foram calculadas de estruturas obtidas de uma otimização geométrica completa, sem qualquer restrição, partindo de estruturas otimizadas inicialmente em nível semi-empírico AM1. Todos os cálculos foram realizados em nível Restricted Hartree-Fock (RHF) usando a base 6-311G(d,p) com o programa Gaussian 98. As estruturas dos confôrmeros mais estáveis em cada caso estão representadas na Figura 1S (Material Suplementar). Foram calculados para cada composto vários rotâmetros, e suas energias RHF estão apresentadas a seguir. Conversão: 1 hartree $=627,5095 \mathrm{kcal} / \mathrm{mol}$.

\section{E(RHF) (hartrees)}

1ca: -616.790157160 e -616.786958059, 1ba: -616.783066710, $616.784654713,-616.785132928$ e -616.785132895 . 2ca: - 
655.831226404 e -655.828432599. 2ba: -655.825791926, $655.827321574,-655.827872655$ e -655.827872649 . 3ca: 500.853370489. 3ba: -500.844660056 . 4ca: -616.793379231 , 616.793408248, -616.794173421 e -616.794173412. 4ba: 616.776030559, -616.780298576, -616.777129804 e -616.776030558. 5ca: $-655.834567746,-655.834678346,-655.835469895$ e 655.835469889. 5ba: $-655.818309976,-655.822819103$ e 655.818305657

\section{CONCLUSÕES}

Inicialmente, através de cálculos computacionais e de técnicas espectroscópicas, como RMN HOM2DJ e ${ }^{13} \mathrm{C}$, determinou-se que as octalonas da série 10-epi-ciperona $\mathbf{1}$ e $\mathbf{2}$ apresentam o ciclo B na conformação cadeira e, conseqüentemente, o substituinte isopropenila em C-7 na posição axial, confirmando resultados prévios da literatura. ${ }^{41}$

Observou-se que, contrariamente ao que ocorre com cicloexanonas bicíclicas saturadas, as enonas bicíclicas não sofrem ataques equatoriais preferencialmente. Em quase todos os casos estudados foi formado majoritariamente o álcool alílico equatorial. O ed mostrou-se altamente dependente de fatores estéricos, mesmo o causado por substituintes em posições remotas, como a isopropenila em C-7.

Finalmente, observou-se que a substituição na dupla ligação conjugada em C-4 leva a uma diminuição da velocidade da reação de adição de hidreto na carbonila. Com $L$-selectride ${ }^{\circledR}$ nesses casos forma-se uma mistura equimolecular dos álcoois epiméricos e com $\mathrm{LiAlH}_{4}$ ocorre uma diminuição importante do $e d$.

\section{MATERIAL SUPLEMENTAR}

Estão disponíveis como Material Suplementar, em http:// quimicanova.sbq.org.br, em arquivo PDF, com acesso livre e gratuito, as estruturas de menor energia calculadas para os compostos 1-5, na Figura 1S. Igualmente, está disponível a Figura 2S - espectro de RMN HOM2DJ do composto $\mathbf{1}$.

\section{AGRADECIMENTOS}

Os autores agradecem em primeiro lugar toda a colaboração prestada em muitos anos pela Prof ${ }^{a}$. Helena Maria Carvalho Ferraz, ${ }^{43}$ que em muito contribuiu para o estabelecimento da linha de pesquisa em Síntese Orgânica em nosso Laboratório na UFRGS. Hoje, essa influência se mantém, e egressos de nosso grupo já desenvolvem seus trabalhos de pesquisa na área em Universidades como a UFBA e a UNIFAL.

Agradecemos ao Grupo de Química Teórica do IQ-UFRGS pelas facilidades computacionais oferecidas e às agências CNPq, FAPERGS e à PROPESQ-UFRGS pelas bolsas e financiamentos concedidos.

\section{REFERÊNCIAS}

1. Ferraz, H. M. C.; Souza, A. J. C.; Tenius, B. S. M.; Bianco, G. G.; Tetrahedron 2006, 62, 9232.

2. Tenius, B. S. M.; de Oliveira, E. R.; Ferraz, H. M. C.; Tetrahedron: Asymmetry 1993, 4, 633.

3. Tenius, B. S. M.; Rohde, A. R.; Victor, M. M.; Viegas, C.; Synth. Commun. 1996, 26, 197.

4. Tenius, B. S. M.; Schroeder, E. K.; Synth. Commun. 2000, 30, 1371.

5. Schenato, R. A.; dos Santos, E. M.; Tenius, B. S. M.; Costa, P. R. R.; Caracelli, I.; Zukerman-Schpector, J.; Tetrahedron: Asymmetry 2001, 12, 579.

6. dos Santos, E. M.; Bogdan, M.; Victor, M. M.; Tenius, B. S. M.; de Oliveira, E. R.; J. Braz. Chem. Soc. 2007, 18, 370.
7. Schenato, R. A.; dos Santos, E. M.; Goncalves, P. F. B.; Tenius, B. S. M.; de Oliveira, E. R.; Caracelli, I.; Zukerman-Schpector, J.; Magn. Reson. Chem. 2003, 41, 53.

8. Schenato, R. A.; dos Santos, E. M.; Tenius, B. S. M.; de Oliveira, E. R.; Quim. Nova 2003, 26, 717.

9. da Silveira, L. G.; Sacheto, D.; Netz, P. A.; de Oliveira, E. R.; J. Mol. Struct. THEOCHEM 2007, 814, 75 .

10. Sauer, A. M.; Crowe, W. E.; Henderson, G.; Laine, R. A.; Tetrahedron Lett. 2007, 48, 6590; Chen, Y. G.; Zhou, G.; Liu, L. J.; Xiong, Z. M.; Li, Y. L.; Synthesis 2001, 1305; Zhang, Z.; Xiong, Z. M.; Zheng, G. J.; Li, Y. L.; Tetrahedron: Asymmetry 2001, 12, 2137; Gao, X. L.; Xiong, Z. M.; Zhou, G.; Li, Y. L.; Synthesis 2001, 37; Zhou, G.; Gao, X. L.; Zhang, Z.; Li, W. D. Z.; Li, Y. L.; Tetrahedron: Asymmetry 2000, 11, 1819; Zhou, G.; Gao, X. L.; Zhang, Z.; Li, Y. L.; J. Chem. Res. (S) 2000, 174; Chen, Y. G.; Xiong, Z. M.; Zhou, G.; Liu, L. J.; Li, Y. L.; Tetrahedron: Asymmetry 1998, 9 , 1923; Zhou, G.; Xiong, Z. M.; Chen, Y. G.; Li, Y. L.; J. Chem. Res. (S) 1998, 650; Xiong, Z. M.; Zhou, G.; Chen, Y. G.; Li, Y. L.; J. Chem. Res. (S) 1998, 450; Chen, Y. G.; Xiong, Z. M.; Zhou, G.; Yang, J.; Li, Y. L.; Chem. Lett. 1997, 1289; Zhabinskii, V. N.; Minnaard, A. J.; Wijnberg, J. B. P. A.; de Groot, A.; J. Org. Chem. 1996, 61, 4022; Xiong, Z. M.; Yang, J.; Li, Y. L.; Tetrahedron: Asymmetry 1996, 7, 2607.

11. Rodenberg, R. E.; Vilardo, J. S.; Tetrahedron Lett. 1996, 37, 2185.

12. Banerjee, A. K.; Vera, W.; J. Chem. Res. (S) 1996, 108.

13. Kim, S. K.; Fuchs, P. L.; J. Am. Chem. Soc. 1993, 115, 5934.

14. Hutchins, R. O.; Su, W. Y.; Sivakumar, R.; Cistone, F.; Stercho, Y. P.; J. Org. Chem. 1983, 48, 3412.

15. D’Incan, E.; Loupy, A.; Maia, A.; Seyden-Penne, J.; Viout, P.; Tetrahedron 1982, 38, 2923.

16. Wigfield, D. C.; Tetrahedron 1979, 35, 449.

17. Vandenheuvel, W. J. A.; Wallis, E. S.; J. Org. Chem. 1962, 27, 1233.

18. Wu, Y. D.; Houk, K. N.; J. Am. Chem. Soc. 1993, 115, 10992.

19. Coxon, J. M.; Luibrand, R. T.; Tetrahedron Lett. 1993, 34, 7097.

20. Frenking, G.; Kohler, K. F.; Reetz, M. T.; Angew. Chem., Int. Ed. 1991, 30, 1146.

21. Wu, Y. D.; Tucker, J. A.; Houk, K. N.; J. Am. Chem. Soc. 1991, 113, 5018.

22. Meyers, A. I.; Wallace, R. H.; J. Org. Chem. 1989, 54, 2509.

23. Wu, Y. D.; Houk, K. N.; J. Am. Chem .Soc. 1987, 109, 906

24. Wu, Y. D.; Houk, K. N.; Trost, B. M.; J. Am. Chem. Soc. 1987, 109, 5560.

25. Cieplak, A. S.; J. Am. Chem. Soc. 1981, 103, 4540.

26. Marshall, J. A.; Ruth, J. A.; J. Org .Chem. 1974, 39, 1971.

27. Kim, S.; Moon, Y. C.; Ahn, K. H.; J. Org. Chem. 1982, 47, 3311.

28. Wilson, K. E.; Seidner, R. T.; Masamune, S.; J. Chem. Soc. Chem. Commun. 1970, 213.

29. Saito, A.; Tanaka, A.; Oritani, T.; Tetrahedron: Asymmetry 1996, 7, 2923.

30. Snitman, D. L.; Tsai, M. Y.; Watt, D. S.; Edwards, C. L.; Stotter, P. L.; J. Org. Chem. 1979, 44, 2838.

31. Brown, H. C.; Krishnamurthy, S.; J. Am. Chem. Soc. 1972, 94, 7159.

32. Brown, H. C.; Krishnamurthy, S.; Hubbard, J. L.; J. Am. Chem .Soc. 1978, $100,3343$.

33. Brown, C. A.; J. Am. Chem. Soc. 1973, 95, 4100.

34. Brown, C. A.; Krishnamurthy, S.; J. Organomet. Chem. 1978, 156, 111.

35. Ganem, B.; J. Org. Chem. 1975, 40, 146.

36. Fortunato, J. M.; Ganem, B.; J. Org. Chem. 1976, 41, 2194.

37. Han, G.; La Porte, M. G.; Folmer, J. J.; Werner, K. M.; Weinreb, S. M.; Angew. Chem., Int. Ed. 2000, 39, 237.

38. Tanis, S. P.; Chuang, Y. H.; Head, D. B.; J. Org. Chem. 1988, 53, 4929.

39. Minnaard, A. J.; Stork, G. A.; Wijnberg, J. B. P. A.; de Groot, A.; J. Org. Chem. 1997, 62, 2344.

40. Frisch, M. J.; Trucks, G. W.; Schlegel, H. B.; Scuseria, G. E.; Robb, M. A.; Cheeseman, J. R.; Zakrzewski, V. G.; Montgomery Jr.; J. A.; Stratmann, R. E.; Burant, J. C.; Dapprich, S.; Millam, J. M.; Daniels, A. D.; Kudin, K. N.; Strain, M. C.; Farkas, O.; Tomasi, J.; Barone, V.; Cossi, M.; Cammi, R.; Mennucci, B.; Pomelli, C.; Adamo, C.; Cliord, S.; Ochterski, J.; Petersson, G. A.; Ayala, P. Y.; Cui, Q.; Morokuma, K.; Malick, D. K.; Rabuck, A. D.; Raghavachari, K.; Foresman, J. B.; Cioslowski, J.; Ortiz, J. V.; Stefanov, B. B.; Liu, G.; Liashenko, A.; Piskorz, P.; Komaromi, I.; Gomperts, R.; Martin, R. L; Fox, D. J.; Keith, T.; Al Laham, M. A.; Peng, C. Y.; Nanayakkara, A.; Gonzalez, C.; Challacombe, M.; Gill, P. M. W.; Johnson, B.; Chen, W.; Wong, M. W.; Andres, J. L.; Gonzalez, C.; HeadGordon, M.; Replogle, E. S.; Pople, J. A.; Gaussian 98, Revision A.9, Gaussian, Inc., Pittsburgh, EUA, 1998.

41. Huffman, J. W.; Swain, W. E.; Jacobus, J.; Mcphail, A. T.; J. Org. Chem. 1980, 45, 3088

42. de Oliveira, E. R.; Victor, M. M.; Ferraz, H. M. C.; Tenius, B. S. M.;

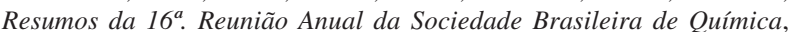
Caxambu, , Brasil, 1993.

43. da Silva, L. F.; Quim. Nova 2007, 30, 1791 


\section{DIASTEREOSSELETIVIDADE NA REDUÇÃO DE ENONAS BICÍCLICAS COM HIDRETOS VOLUMOSOS}

Andreza C. Camozzato, Beatriz S. M. Tenius e Eduardo R. de Oliveira*

Departamento de Química Orgânica, Instituto de Química, Universidade Federal do Rio Grande do Sul, CP 15003, 91501-970 Porto Alegre - RS, Brasil

Cláudio Viegas Jr.

Departamento de Farmácia, Universidade Federal de Alfenas, Rua Gabriel Monteiro da Silva, 714, 37130-000 Alfenas - MG, Brasil Maurício M. Victor

Departamento de Química Orgânica, Instituto de Química, Universidade Federal da Bahia, Campus de Ondina, 40170-115

Salvador - BA, Brasil

Leandro G. da Silveira

Departamento de Ciências Exatas e da Terra, Curso de Química, Universidade Regional Integrada do Alto Uruguai e das Missões - Campus de Frederico Westphalen, CP 184, 98400-000 Frederico Westphalen - RS, Brasil

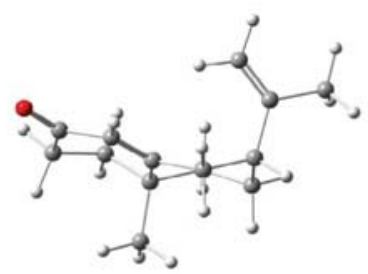

$1 \mathrm{ca}$

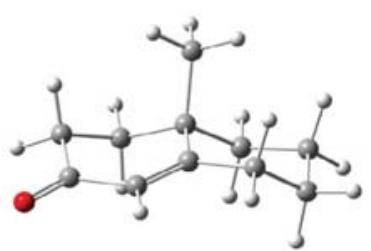

3ca

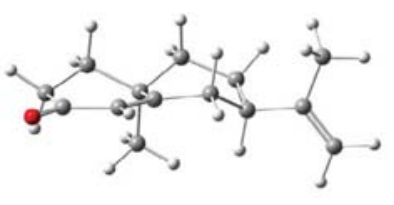

1 ba

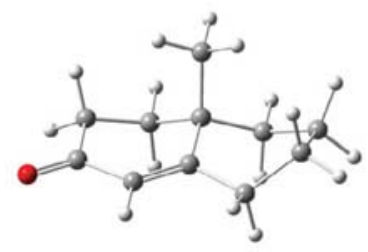

3ba

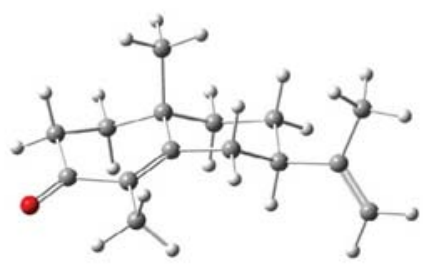

$5 \mathrm{ca}$

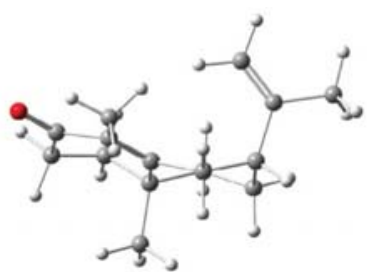

2ca

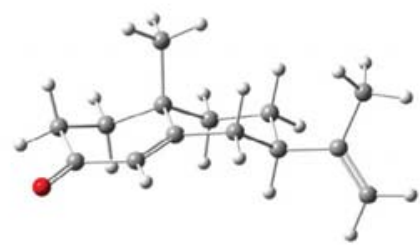

4ca

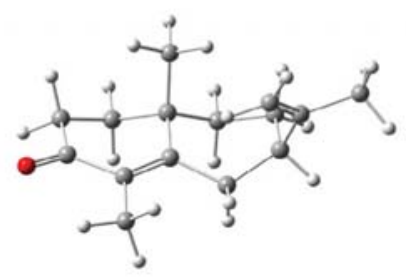

5 ba

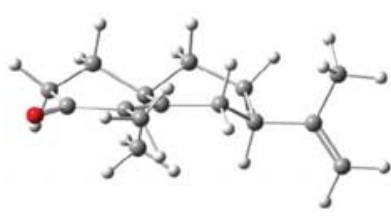

2ba

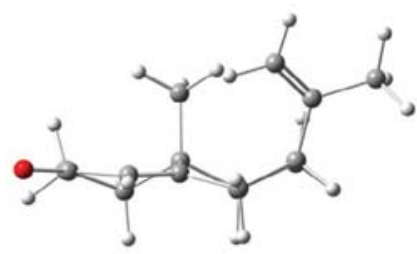

4ba

Figura 1S. Estruturas de menor energia calculadas para os compostos 1-5. Confôrmero cadeira para o ciclo B - ca; barco torcido - ba 


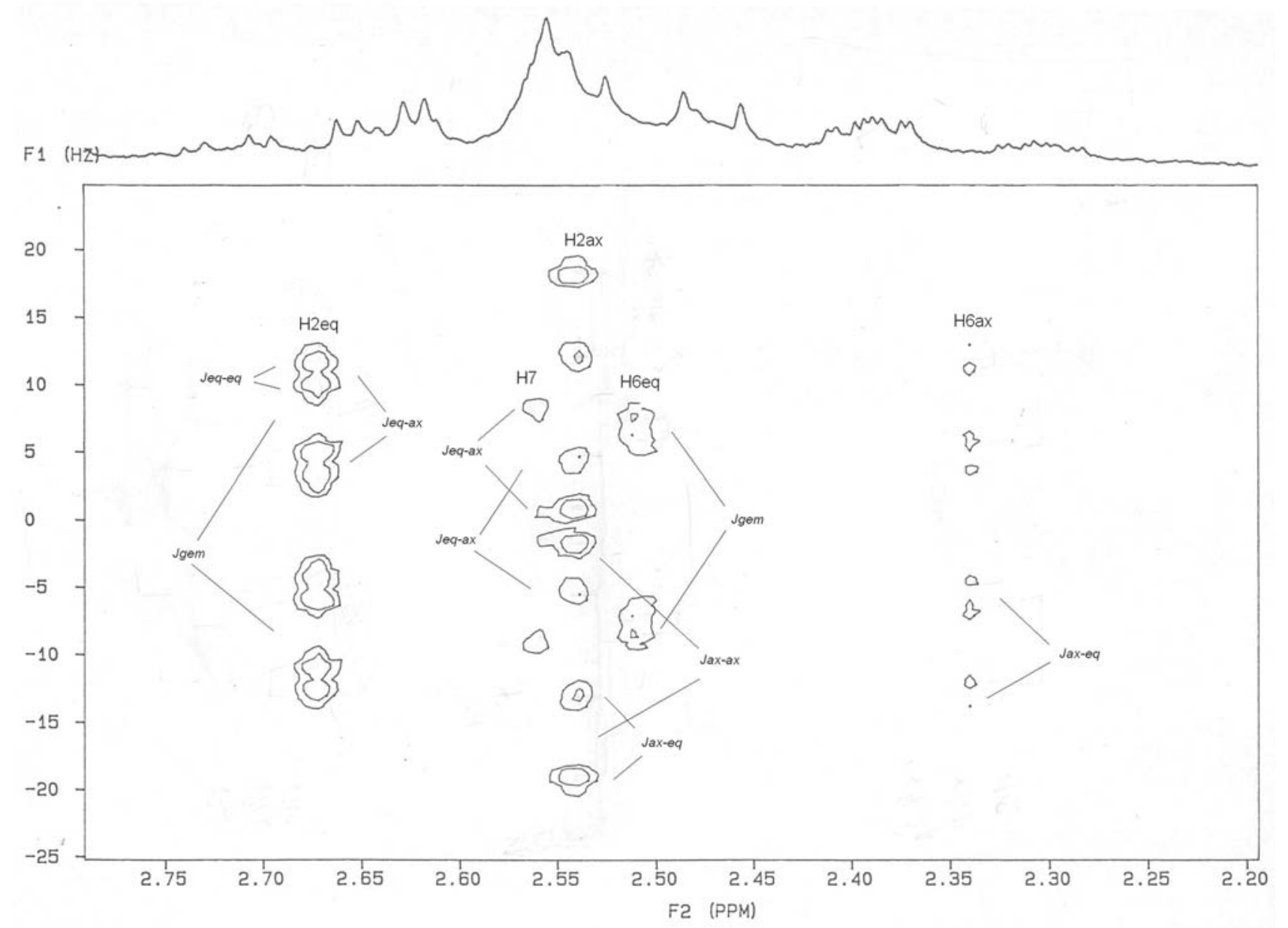

Figura 2S. Espectro de RMN HOM2DJ do composto 1 